\title{
Approach to evaluating tumor mutational burden in routine clinical practice
}

\author{
John Truesdell, Vincent A. Miller, David Fabrizio \\ Foundation Medicine, Inc., Cambridge, MA, USA \\ Contributions: (I) Conception and design: All authors; (II) Administrative support: None; (III) Provision of study materials or patients: None; (IV) \\ Collection and assembly of data: All authors; (V) Data analysis and interpretation: All authors; (VI) Manuscript writing: All authors; (VII) Final \\ approval of manuscript: All authors. \\ Correspondence to: Vincent A. Miller, MD. Foundation Medicine, Inc., 150 Second Street, Cambridge, MA 02141, USA. \\ Email: vmiller@foundationmedicine.com.
}

\begin{abstract}
Immune checkpoint inhibition with monoclonal antibodies has emerged as a promising therapeutic approach but in most tumor types responses are unpredictable and observed in a minority of treated patients. Positive and negative predictive biomarkers for efficacy of these costly drugs are desperately needed. Immunohistochemistry (IHC) for programmed death ligand (PD-L1) expression in tumor and inflammatory infiltrate has emerged as one predictive biomarker of some value. However, multiple confounders including those inherent to any IHC and the unique complexities of the biology of the immune response have limited its utility. Tumor mutational burden (TMB) has emerged as a seemingly more promising predictive biomarker for immunotherapy with checkpoint inhibitors in several tumor types and is likely to be incorporated into future treatment algorithms for these agents. Given this, the need to define and standardize key parameters of the most promising biomarkers becomes essential to allow all stakeholders to make meaningful observations and inferences as to the efficacy of ostensibly similar agents and combinations in various settings. This review briefly summarizes approaches to measurement of TMB and ongoing efforts to achieve harmonization of this key biomarker.
\end{abstract}

Keywords: Tumor mutational burden (TMB); non-small cell lung cancer (NSCLC); comprehensive genomic profiling (CGP)

Submitted Aug 16, 2018. Accepted for publication Sep 26, 2018.

doi: $10.21037 /$ tlcr.2018.10.10

View this article at: http://dx.doi.org/10.21037/tlcr.2018.10.10

\section{Introduction}

Tumor mutational burden (TMB) has emerged as a promising predictive biomarker for immunotherapy with checkpoint inhibitors in non-small cell lung cancer (NSCLC) and other tumor types. While whole exome sequencing (WES) is widely considered the gold standard for measurement of TMB, performance of WES from tumors of patients with cancer is currently impractical for multiple reasons. Comprehensive genomic profiling (CGP) based on next-generation sequencing (NGS) technology has offered a practical estimate of TMB from the whole exome in the clinical setting. Checkpoint inhibitors are currently being evaluated in several thousand trials both alone and in combination, covering nearly all tumor types. The vast majority of these incorporate both established and exploratory correlative biomarker studies for prediction of response and are more commonly phase I or II studies treating dozens not $100 \mathrm{~s}$ of patients. Given this, the need to define and standardize key parameters of the most promising biomarkers becomes essential to allow all stakeholders to make meaningful observations and inferences as to the efficacy of ostensibly similar agents and combinations in various settings. This review briefly summarizes approaches to measurement of TMB and ongoing efforts to achieve harmonization of this key biomarker. 


\section{Background and rationale for studying TMB}

The response rates for checkpoint inhibitor monotherapies remain low (approximately 20\%) in unselected patient populations in most cancer types, and the cost of these therapies remains high, at approximately $\$ 150,000$ per patient per year (1). Therefore, biomarkers that predict which patients are most likely to benefit from immune checkpoint inhibitors are a critical unmet medical need for the clinical management of patients with cancer. Unfortunately, identifying patients whose tumors will respond to immunotherapy has proved challenging. Programmed death ligand (PD-L1) immunohistochemistry (IHC) has become a standard of care for categorizing patients who may benefit from immunotherapy. However, PD-L1 IHC has a number of weaknesses including: multiple antibody clones utilized in clinical trials, such as the Dako 22C3, Dako 28-8 and the Ventana SP142, varied scoring methodologies [e.g., tumor proportion score (TPS) and combined positive score (CPS), with Dako 22C3] and inter- and intra-tumor, pathologist and lab variability. Aside from the technical caveats, a "positive" PD-L1 result does not always provide an effective stratification of patients based on response to immunotherapy.

Efficacy of checkpoint inhibitors is predicated on many host, tumor and microenvironment characteristics, some of which remain incompletely understood. However, the hypothesis that increased TMB is correlative with an increased probability of expressing a sufficient number of immune reactive neo-antigens, which are necessary but not always sufficient for efficacy of checkpoint inhibition is not new and at least in part, this theory has been tested clinically by evaluating TMB as a continuous variable $(2,3)$. Additionally, a number of studies have now shown that higher TMB is associated with better outcomes across a number of tumor types including NSCLC, colorectal cancer (CRC), bladder and melanoma, among others (4-15).

\section{Assessment and reporting of TMB}

The initial standard approach for assessing TMB involved WES analysis of matched tumor tissue and normal specimens using NGS $(5,7,13,14)$. However, WES requires high coverage sequencing of $\sim 50 \mathrm{Mb}$ of genomic content, and thus, is cost prohibitive for routine clinical use in oncology at present. Additionally, the current standard turnaround-time for WES analysis is 4-6 weeks due to the intense sequencing and analyses required. As a result, targeted NGS panels to assess TMB have been developed, which are more cost effective and have a lower turn-around time than WES. Several studies have explored the use of NGS panels rather than WES to assess different diseases including NSCLC (2), melanoma (11), bladder cancer (12), hyper-mutated pediatric glioblastoma (15), CRC (10), and a broader pan-tumor study that demonstrated retrospective clinical validity across more than 20 tumor types (3).

Variability in calculating TMB can arise from different platforms that have unique targeted panels and technologies, therefore it is critical to harmonize methods for TMB calculation and reporting by establishing clear standardization guidelines that incorporate consistent methods that describe both the analytical validation requirements and suggest consistent methods for establishing clinical validation. TMB is derived from CGP utilizing a sophisticated algorithm to account for the smaller subset of the exome assessed. For example, Foundation Medicine incorporates both nonsynonymous and synonymous base substitutions and short insertion and deletion alterations in the calculation. Synonymous base substitutions are assumed not to generate neo-antigens, but when correlated with nonsynonymous base substitutions improve sensitivity and are potentially reflective of a more immunogenically reactive tumor state. Additionally, known somatic alterations in the COSMIC database, truncations in tumor suppressor genes and germline alterations are also filtered out to reduce bias. This approach for calculating TMB has been reviewed by Food and Drug Administration as part of the FoundationOne CDx platform and approved as an analytic claim (16).

CGP utilizing a broad panel, such as FoundationOne CDx, has demonstrated high statistical concordance with TMB measured from WES. An analysis was conducted using a direct comparison of TMB between WES and the Foundation One $\mathrm{CDx}$, which uses approximately $0.8 \mathrm{Mb}$ of coding region to calculate TMB, on 44 NSCLC specimens from CheckMate-026. Results from a scatter plot of TMB values between WES- and CGP-derived TMB yielded a Pearson's correlation of 0.95 and an overall agreement in the TMB calculation based on the cutoff of 10 mutations/Mb of $86 \%$ (17). Furthermore, Chalmers and colleagues reported that a key determinant for the accuracy of the CGP-based TMB measurement is the number of megabases $(\mathrm{Mb})$ sequenced in the genome, and that sequencing approximately $1.1 \mathrm{Mb}$ across 315 genes resulted in a TMB estimate that was similar to the reference standard of WES $\left(\mathrm{R}^{2}=0.74\right)(18)$. The study also estimated 
that sampling approximately $0.5 \mathrm{Mb}$ or less resulted in an unacceptable degree of difference from the WES reference standard, suggesting that more limited assays may result in an inaccurate TMB calculation.

As there are a number of CGP assays that measure TMB currently marketed or under development, validation across multiple dimensions will be critical to establish the analytical performance and clinical utility, allowing improved interpretation of clinical trial results and agents, which appear to be mechanistically quite similar. In silico accuracy against WES should not be considered sufficient, but other performance metrics such as precision of the TMB cutoff, limit of detection according to the minimum tumor purity, and empirical accuracy against a gold standard, such as WES, should be established for any TMB platform currently being evaluated as a companion diagnostic. In addition, alignment against a universal set of reference standards will ensure consistency of calculating TMB across platforms and should be considered an essential parameter of an analytic validation plan.

TMB has emerged as a biomarker that is distinct from PD-L1 IHC and addresses many of its shortcomings. TMB's inherent advantages arise from its quantitative nature, which avoids the need for human interpretation and can be standardized, avoiding the confusion of antibody choice and interpretation variability. Also, to avoid the same weaknesses the befell PD-L1 IHC, there are efforts by Friends of Cancer Research, the FDA, and key industry leaders to agree on a standard methodology to assess and report TMB (19).

\section{Steps for the harmonization of TMB: analytic and clinical validation}

Harmonization of TMB requires agreeing on best practices that establish consistent performance through analytic validation studies, which at a minimum require demonstrating accuracy, precision, and limit of detection. An additional essential component includes alignment of the TMB calculation against a universal set of reference standards to ensure consistency of reporting across different technological platforms. In order to promote harmonization around TMB analytic validation and reference standard alignment, a consortium of industry, academia, policy makers and regulatory agencies, including the FDA, led by Friends of Cancer Research aims to propose best practices by publishing industry accepted standards. The aim of the consortium is to develop a consistent methodology and to publish these methodologies prior to approval of TMB as a companion diagnostic, which will accelerate the broad adoption of this biomarker and further necessitate standardization requirements. Ultimately, the findings obtained by the consortium will shape the requirements for demonstrating analytic validation of TMB, guide the clinical application and inform the development of prospective studies where TMB is used as a companion diagnostic.

\section{Acknowledgements}

None.

\section{Footnote}

Conflicts of Interest: The authors have no conflicts of interest to declare.

\section{References}

1. Kohn CG, Zeichner SB, Chen Q, et al. Cost-Effectiveness of Immune Checkpoint Inhibition in BRAF Wild-Type Advanced Melanoma. J Clin Oncol 2017;35:1194-202.

2. Gandara DR, Paul SM, Kowanetz M, et al. Bloodbased tumor mutational burden as a predictor of clinical benefit in non-small-cell lung cancer patients treated with atezolizumab. Nat Med 2018;24:1441-8.

3. Goodman AM, Kato S, Bazhenova L, et al. Tumor Mutational Burden as an Independent Predictor of Response to Immunotherapy in Diverse Cancers. Mol Cancer Ther 2017;16:2598-608.

4. Hellmann MD, Ciuleanu TE, Pluzanski A, et al. Nivolumab plus Ipilimumab in Lung Cancer with a High Tumor Mutational Burden. N Engl J Med 2018;378:2093-104.

5. Le DT, Uram JN, Wang H, et al. PD-1 Blockade in Tumors with Mismatch-Repair Deficiency. N Engl J Med 2015;372:2509-20.

6. Le DT, Durham JN, Smith KN, et al. Mismatch repair deficiency predicts response of solid tumors to PD-1 blockade. Science 2017;357:409-13.

7. Rizvi NA, Hellmann MD, Snyder A, et al. Cancer immunology. Mutational landscape determines sensitivity to PD-1 blockade in non-small cell lung cancer. Science 2015;348:124-8.

8. Overman MJ, Lonardi S, Wong KYM, et al. Durable Clinical Benefit With Nivolumab Plus Ipilimumab in DNA Mismatch Repair-Deficient/Microsatellite Instability-High 
Metastatic Colorectal Cancer. J Clin Oncol 2018;36:773-9.

9. Overman MJ, McDermott R, Leach JL et al. Nivolumab in patients with metastatic DNA mismatch repair-deficient or microsatellite instability-high colorectal cancer (CheckMate 142): an open-label, multicentre, phase 2 study. Lancet Oncol 2017; 18:1182-1191.

10. Fabrizio DA, George TJ Jr, Dunne RF, et al. Beyond microsatellite testing: assessment of tumor mutational burden identifies subsets of colorectal cancer who may respond to immune checkpoint inhibition. J Gastrointest Oncol 2018;9:610-7.

11. Johnson DB, Frampton GM, Rioth MJ, et al. Targeted Next Generation Sequencing Identifies Markers of Response to PD-1 Blockade. Cancer Immunol Res 2016;4:959-67.

12. Rosenberg JE, Hoffman-Censits J, Powles T, et al. Atezolizumab in patients with locally advanced and metastatic urothelial carcinoma who have progressed following treatment with platinum-based chemotherapy: a single-arm, multicentre, phase 2 trial. Lancet 2016;387:1909-20.

13. Snyder A, Makarov V, Merghoub T, et al. Genetic basis for clinical response to CTLA-4 blockade in melanoma. N Engl J Med 2014;371:2189-99.

14. Van Allen EM, Miao D, Schilling B, et al. Genomic

Cite this article as: Truesdell J, Miller VA, Fabrizio D. Approach to evaluating tumor mutational burden in routine clinical practice. Transl Lung Cancer Res 2018;7(6):678-681. doi: $10.21037 /$ tlcr.2018.10.10 correlates of response to CTLA-4 blockade in metastatic melanoma. Science 2015;350:207-11.

15. Campbell BB, Light N, Fabrizio D, et al. Comprehensive Analysis of Hypermutation in Human Cancer. Cell 2017;171:1042-56.e10.

16. FoundationOne CDx FDA approval. Nov 30, 2017. Available online: https://www.fda.gov/ medicaldevices/productsandmedicalprocedures/ deviceapprovalsandclearances/recently-approveddevices/ ucm590331.htm

17. Szustakowski JD, Green G, Geese WJ, et al. Evaluation of tumor mutation burden as a biomarker for immune checkpoint inhibitor efficacy: a calibration study of whole exome sequencing with FoundationOne ${ }^{\circledR}$ (abstract). Chicago, Illinois: AACR Annual Meeting, 2018. Available online: http://www.abstractsonline.com/pp8/\#!/4562/ presentation $/ 2718$

18. Chalmers ZR, Connelly CF, Fabrizio D, et al. Analysis of 100,000 human cancer genomes reveals the landscape of tumor mutational burden. Genome Med 2017;9:34.

19. Karow J. Two initiatives seek to harmonize tumor mutational burden testing. Available online: https://www. genomeweb.com/cancer/two-initiatives-seek-harmonizetumor-mutational-burden-testing\#.W87uF2gzbIU 